Rev. Adm. Saúde (On-line), São Paulo, v. 19, n. 74, jan. - mar. 2019, Epub 18 mar. 2019 http://dx.doi.org/10.23973/ras.74.150

ARTIGO DE ATUALIZAÇÃO

\title{
Desafios atuais dos laboratórios de saúde pública frente às novas exigências regulatórias
}

Current challenges of public health laboratories with the new regulatory requirements

\section{Blanca Elena Ortega Markman', Ellen Gameiro Hilinski², Fernanda Fernandes Farias ${ }^{3}$}

1. Farmacêutica, mestre em fármacos e medicamentos. Pesquisador científico do Instituto Adolfo Lutz. São Paulo SP

2. Farmacêutica. Pesquisador científico do Instituto Adolfo Lutz. São Paulo SP

3. Farmacêutica. Pesquisador científico do Instituto Adolfo Lutz. São Paulo SP

\section{RESUMO}

O avanço regulatório, observado no mercado farmacêutico brasileiro nas últimas décadas, representa um impacto positivo para a população, na medida em que passa a dispor de medicamentos com melhores perfis de segurança e eficácia, reduzindo e prevenindo o risco inerente às propriedades farmacológicas e toxicológicas dos fármacos. Este estudo visa atualizar o panorama da qualidade de medicamentos no Brasil e os desafios enfrentados pelos laboratórios de saúde pública para a condução dos ensaios analíticos de monitoramento da qualidade. Com o incremento do lançamento de novas tecnologias farmacêuticas, como os biofármacos, e a publicação de nova regulamentação, como a RDC 53/2015, o atendimento às novas exigências dos órgãos regulatórios impõem desafios complexos para os laboratórios oficiais. A notificação, identificação e qualificação de produtos de degradação em medicamentos com substâncias ativas sintéticas e semissintéticas, bem como a análise do polimorfismo e de fármacos quirais, são alguns dos exemplos que 
demandam o desenvolvimento e validação de técnicas analíticas com maior seletividade e complexidade. Para efetivar este avanço torna-se essencial a utilização de equipamentos robustos e modernos, assim como a capacitação do corpo analítico nos laboratórios de saúde pública.

Palavras-chave: monitoramento da qualidade, medicamentos, laboratório de saúde pública, regulamentação.

\section{ABSTRACT}

The regulatory improvement observed in the Brazilian pharmaceutical market on the last decades represents a positive impact for the population which now has medicines with better safety and efficacy profiles, reducing and preventing the inherent risk in the pharmacological and toxicological drug properties. This study aims to update the scenery of the quality of medicines in Brazil and the challenges faced by public health laboratories to conduct the analytical tests to quality assurance. With the increase in the launching of new pharmaceutical technologies, such as biopharmaceuticals and the publication of new regulations, such as RDC 53/2015, meet the new regulatory requirements imposes complex challenges for official laboratories. The notification, identification and qualification of degradation products in pharmaceutical products with synthetic and semisynthetic active substances, as well the analysis of polymorphism and chiral drugs are some of the examples that demand the development and validation of analytical techniques with greater selectivity and complexity. To achieve this advance, the use of robust and modern equipment is essential, as well as the training of the analytical group in the public health laboratories.

Keywords: quality monitoring, medicines, public health laboratory, regulation

\section{INTRODUÇÃO}

A disponibilidade de medicamentos efetivos e seguros, como um dos principais diferenciadores entre a prática da medicina atual e das últimas décadas, constitui um importante instrumento de saúde que visa interromper o processo de adoecimento e redução das taxas de mortalidade e morbidade, bem como a melhoria da qualidade de vida dos indivíduos. ${ }^{1}$

O aumento da incidência de doenças crônicas, do envelhecimento da população e da disseminação de novas e custosas tecnologias médicas vêm contribuindo com o aumento acentuado do consumo de medicamentos e de serviços de saúde em países desenvolvidos e em desenvolvimento, resultando em impacto significativo nos gastos públicos. ${ }^{2}$ 
Como resultado deste cenário, a partir da década de 80 , amplas reformas sanitárias foram iniciadas em distintos países, incluindo o Brasil, com o objetivo de reorganizar os sistemas de saúde e as atividades relacionadas à farmacoterapia. $^{2}$

Em 1998 foi instituída a Política Nacional de Medicamentos no Brasil com o objetivo de garantir a segurança, eficácia e qualidade dos medicamentos, através da regulação sanitária, assistência farmacêutica na promoção da pesquisa, desenvolvimento e produção de insumos e medicamentos categorizados como essenciais. ${ }^{3}$ Como ações desta política, ocorreu a implementação do programa de medicamentos genéricos, a revisão da legislação que regulamenta a propaganda de medicamentos em suas diversas formas, a elaboração do formulário terapêutico nacional contendo a Relação Nacional de Medicamentos Essenciais, além da melhoria ao acesso pela população aos medicamentos essenciais. ${ }^{4}$

Com a criação da Agência Nacional de Vigilância Sanitária (ANVISA), através da Lei 9.782/1999, cuja missão consiste em proteger e promover a saúde da população com o intuito de garantir a segurança sanitária de produtos e serviços, o avanço regulatório tornou-se efetivo por meio do conjunto de normas estabelecidas pela autoridade sanitária no âmbito das definições da política de medicamentos. ${ }^{5}$

A implantação dos medicamentos genéricos no mercado brasileiro, por meio da Lei № 9787/1999, consagrou-se como uma experiência de sucesso para a integração das políticas sociais voltadas ao maior acesso da população à produtos farmacêuticos de qualidade e à intercambialidade das apresentações, conforme preconizado pela Organização Mundial de Saúde. Tal medida contribuiu para o desenvolvimento do panorama da qualidade dos medicamentos, com consequente intensificação no monitoramento das unidades produtivas e ao atendimento às Boas Práticas de Fabricação após a publicação da Resolução da Diretoria Colegiada (RDC) no 17/2010 da ANVISA. $6-8$

Devido a necessidade de monitorar o mercado farmacêutico brasileiro com relação às queixas técnicas dos produtos e às reações adversas dos fármacos, a ANVISA implementou um sistema de farmacovigilância embasado em antecedentes históricos dos produtos e que resultou no avanço da avaliação da qualidade de medicamentos. ${ }^{9}$ Atualmente, este sistema é parte integrada, obrigatória entre as diversas áreas da cadeia farmacêutica e se tornou indispensável ao monitoramento das reações adversas, desvios de qualidade identificados na etapa de pós-comercialização, além de disponibilizar informações isentas do viés mercadológico para prescritores e consumidores. ${ }^{9}$

Para cumprir com as exigências relativas aos ensaios de equivalência farmacêutica e bioequivalência, após a introdução da política de medicamentos genéricos, foi necessário o desenvolvimento de uma nova infraestrutura de pesquisa e desenvolvimento técnico e de capacitação pelas indústrias farmacêuticas, bem como pelas instituições públicas de saúde. ${ }^{10}$ 
Nesse sentido, análises comparativas entre os medicamentos genéricos e de referência, como os estudos de bioequivalência, estudos fármacodinâmicos comparativos em humanos, testes clínicos comparativos e testes in vitro, sendo o principal o ensaio de dissolução, demandaram novos investimentos por parte da cadeira produtora. ${ }^{8}$

A certificação da infraestrutura do Instituto Nacional de Controle de Qualidade em Saúde (INCQS/Fiocruz) e dos Laboratórios Centrais de Saúde Pública (LACENS) teve como objetivo obter o suporte laboratorial e a capacitação para a participação em programas relativos à verificação da qualidade dos medicamentos disponíveis no mercado brasileiro. ${ }^{11}$

Desta maneira a ANVISA e o INCQS instituíram o Programa Nacional de Verificação da Qualidade de Medicamentos (PROVEME) de modo a implantar um programa nacional de análise laboratorial de medicamentos visando monitorar a qualidade desses produtos, promover o saneamento do mercado e construir um modelo de intervenção preventiva, ou seja, antes de haver dano ou agravo à saúde da população. ${ }^{11}$

\section{OBJETIVO}

Este estudo visa pontuar as dificuldades encontradas pelos laboratórios de saúde pública brasileiros durante as atividades de monitoramento da qualidade de medicamentos frente às novas legislações e às tecnologias intrínsecas de novos produtos farmacêuticos.

\section{METODOLOGIA}

Foi realizada uma ampla pesquisa em dados da literatura tanto de periódicos nacionais quanto internacionais nas principais bases de dados disponíveis na internet, como Scielo, PubMed, Scopus. Utilizaram-se como palavras-chaves: laboratórios públicos, medicamentos genéricos, biofármacos, produtos de degradação, desafios da indústria farmacêutica, regulamentação de fármacos. Os artigos foram selecionados conforme relevância ao tema e atualidade das informações. Como literatura adicional também foram consultadas as regulamentações da área, guias e informações técnicas de órgãos oficiais.

\section{RESULTADOS E DISCUSSÃO}

Com a expiração do prazo de vigência de distintas patentes no mercado brasileiro, as indústrias farmacêuticas intensificaram os investimentos em pesquisa e desenvolvimento de novos produtos. ${ }^{12}$

Atualmente, verifica-se uma tendência pela formação de conglomerados da indústria farmacêutica para o desenvolvimento e produção de biofármacos como investimento prioritário, uma vez que dos US\$ 707 bilhões das vendas 
globais em 2010, US $\$ 130$ bilhões estiveram relacionados a produtos de base tecnológica (18,4\%). Estima-se que até 2020 este grupo seja responsável por US $\$ 250$ bilhões das vendas. Drogas biotecnológicas estão se tornando foco de investimentos das indústrias inovadoras, mercado considerado menos exposto à concorrência e cuja produção, é em tese, mais difícil de ser reproduzida. ${ }^{12}$

O maior objetivo dos biofármacos está relacionado com o tratamento e prevenção de numerosas doenças raras e graves, incluindo doenças como câncer, infarto agudo do miocárdio, acidente vascular cerebral, esclerose múltipla, diabetes, artrite reumatóide e doenças autoimunes. Um maior acesso dos pacientes a tratamentos de custo elevado por meio dos medicamentos biossimilares desenvolvidos resulta em melhores respostas terapêuticas, uma vez que apresentam maior especificidade. ${ }^{13}$

Diferentemente dos medicamentos sintéticos produzidos por síntese química, os biofármacos são produzidos por processos de bioengenharia envolvendo bactérias, leveduras ou células animais. Isso faz com que sejam extremamente sensíveis aos métodos de produção, podendo sofrer modificações diante de condições físicas, como a temperatura e a luz. Desta forma as etapas para o desenvolvimento e produção de biofármacos precisam ser rigorosamente monitoradas e avaliadas. ${ }^{14}$

Como incentivo às empresas nacionais, o Governo Federal tem adotado medidas a fim de viabilizar a infraestrutura, a regulamentação e o aparato jurídico necessário. Assim realizam parcerias com os laboratórios privados, nacionais ou internacionais, detentores das patentes dos produtos biológicos, de modo que transfiram a tecnologia para os laboratórios públicos nacionais, até que estes tenham independência na produção. Em troca, o governo garante a compra do medicamento produzido internamente durante o período de transferência de tecnologia, estipulado em, no máximo, cinco anos. Esses acordos devem gerar uma economia de $\mathrm{R} \$ 5,3$ bilhões por ano em compras públicas. ${ }^{15}$ A pesquisa de novos excipientes e de suas aplicações estratégicas ofereceu uma oportunidade inovadora de aumentar a funcionalidade e o desempenho no que diz respeito à administração e a farmacocinética do medicamento. Assim a evolução das novas apresentações farmacêuticas, como as de liberação modificada, os medicamentos nanotecnológicos e os medicamentos biológicos, inferiram em novos desafios analíticos aos laboratórios de saúde pública. ${ }^{14,16}$

Aliado a este novo cenário, a publicação da RDC n‥53, em 2015, estabeleceu parâmetros para a notificação, identificação e qualificação de produtos de degradação em medicamentos com substâncias ativas sintéticas e semissintéticas. A ANVISA instituiu o prazo até dezembro de 2020 para que as indústrias farmacêuticas atendam à nova legislação, para aqueles produtos que já se encontram no mercado. ${ }^{17}$

Desta forma, o atendimento às novas exigências dos órgãos regulatórios, referente à avaliação de compostos residuais de síntese ou de degradação, bem como a análise do polimorfismo e de fármacos quirais, são alguns dos exemplos de dificuldades encontradas no estabelecimento de técnicas 
analíticas seletivas e complexas para executar o monitoramento da qualidade dos medicamentos.

Para os laboratórios de saúde pública, os ensaios analíticos relacionados aos produtos de degradação de produtos farmacêuticos, são, portanto, um desafio para os analistas, uma vez que se faz necessária à utilização de instrumental moderno e capacitação técnica constante para atender às novas exigências regulatórias. ${ }^{17}$

Em 2018, a Farmacopeia Americana aprovou os novos capítulos gerais 232 e 233, os quais estabeleceram, respectivamente, os limites de impurezas elementares e as técnicas analíticas indicadas para a determinação individual de metais pesados em produtos farmacêuticos, matérias primas e medicamentos. ${ }^{18}$

Estas análises também se constituem como desafios analíticos, uma vez que exigem conhecimentos específicos na técnica de Espectrômetro de Emissão Óptica em Argônio Induzido à Plasma, bem como a validação da metodologia analítica. Por meio desta ferramenta é possível o desenvolvimento de métodos para o controle quantitativo de metais pesados, o qual já está previsto no documento Q3D Guideline for elemental impurities publicado pela International Conference on Harmonisation desde 2015), com prazo de implantação de 36 meses. ${ }^{19}$

Apesar de no Brasil ainda não ter sido publicado nenhuma resolução que torne obrigatória a determinação de metais pesados na apresentação final dos medicamentos, muitos laboratórios privados já estão se preparando para tal exigência. Certamente este será o próximo desafio enfrentado pelos laboratórios de saúde pública.

De acordo com o trabalho realizado por Farias et. al (2010), a qualidade dos medicamentos distribuídos a população tem melhorado ao longo dos anos. Não somente pela implementação de programas de monitoramento, mas também pela efetivação das Boas Práticas de Fabricação. ${ }^{20}$

De modo a acompanhar a evolução do mercado farmacêutico, é imprescindível a capacitação continuada da equipe técnica dos LACENS assim como a incorporação de tecnologias capazes de identificar e quantificar os produtos de degradação, surgindo assim a necessidade de sua implementação para garantir a segurança de uso dos medicamentos. ${ }^{20}$

No intuito de contribuir para o monitoramento da qualidade de medicamentos, programas estaduais municipais e federias são instituídos em laboratórios oficiais de saúde pública. ${ }^{21-23}$

Recentemente, o Instituto Adolfo Luz (IAL) participou do PROVEME, entre outros programas que analisam produtos relacionados à saúde da mulher e saúde mental. Outra atividade também executada pelos laboratórios oficiais é a avaliação das queixas técnicas registradas junto aos grupos de Vigilância Sanitária regionais, que vem apresentando características diversificadas com o passar dos anos. 
Se em períodos anteriores a falta de eficácia e a falsificação de medicamentos constituíam as principais reclamações, hoje as reações adversas são a grande maioria, podendo estar relacionadas à presença de produtos de degradação, compostos quirais e à presença de polimorfos não desejados.

Considerando este ponto, o desenvolvimento de novas metodologias analíticas, como a que emprega o uso de cromatografia liquida de alta pressão acoplada a espectrometria de massas, passa a ser uma poderosa ferramenta. Esta permite caracterizar as moléculas pela medida da relação massa/carga dos íons e, portanto, avaliar e monitorar a presença de produtos de degradação e garantir a qualidade de produtos neste novo cenário, podendo ser empregada em todo o ciclo de desenvolvimento, produção e estabilidade do medicamento. ${ }^{24}$

Com o investimento em novos fármacos por parte da indústria farmacêutica com biotecnologia agregada, além do aprimoramento das regulamentações que exigem cada vez mais qualidade e segurança aos medicamentos, surge a necessidade de se utilizar técnicas analíticas mais específicas e robustas para a verificação e monitoramento da qualidade destes medicamentos.

Para a execução destas análises de alta complexidade é necessário que se forneçam continuamente subsídios para a capacitação do corpo técnico, de modo a atender os constantes desafios analíticos.

\section{CONSIDERAÇÕES FINAIS}

O avanço regulatório representa um impacto positivo para a população, na medida em que aumenta a competitividade, que passará a dispor de medicamentos com melhores perfis de segurança e eficácia, reduzindo e prevenindo o risco inerente às propriedades farmacológicas e toxicológicas dos fármacos.

Sendo o estado responsável por monitorar e regular toda a cadeia produtiva até a sua administração, é de suma importância que o mesmo promova uma reestruturação dos programas de monitoramento para avaliar o impacto dos produtos de degradação previstos na RDC no 53/2015, bem como uma atualização das monografias dos compêndios oficiais. ${ }^{16}$

Da mesma forma que são essenciais os subsídios para a formação de complexo tecnológicos nos laboratórios de saúde pública, assim como a capacitação do corpo técnico na execução de análises modernas.

\section{REFERÊNCIAS}

1. Jacobzone S. Pharmaceutical policies in OECD countries: reconciling social and industrial goals. OECD Labour Market and Social Policy Occasional Papers. Paris: OECD Publising, 2000. 
2. Rego ECL. Políticas de regulação do mercado de medicamentos: a experiência internacional. Revista do BNDES. 2000;7(14):367-400. Disponível em: http://web.bndes.gov.br/bib/jspui/handle/1408/11522.

3. Junior DMP, et al. A definição de medicamentos prioritários para o monitoramento da qualidade laboratorial no Brasil: articulação entre a vigilância sanitária e a Política Nacional de Medicamentos. Cad Saúde Pública. 2008; 24(9): 2081-2090. DOI: 0.1590/S0102-311X2008000900014

4. Brasil. Secretaria de Políticas de Saúde. Política Nacional de Medicamentos. Portaria n. 3.916/GM, 30 outubro de 1998. Diário Oficial da União no.15-E, Seção I, pág. 18 a 22, 10 novembro de 1998.

5. Brasil. Casa Civil. Define o Sistema Nacional de Vigilância Sanitária, cria a Agência Nacional de Vigilância Sanitária, e dá outras providências. Lei $n$. 9.782, de 26 de janeiro de 1999. Diário Oficial da União, Seção I, pág. 1, 27 de janeiro de 1999.

6. Brasil. Casa Civil. Altera a Lei no 6.360, de 23 de setembro de 1976, que dispõe sobre a vigilância sanitária, estabelece o medicamento genérico, dispõe sobre a utilização de nomes genéricos em produtos farmacêuticos e dá outras providências. Lei n. 9787 de 10 de fevereiro de 1999. Diário Oficial da União, Seção I, pag. 1, 11 de fevereiro de 1999.

7. Brasil. Ministério da Saúde. Agência Nacional de Vigilância Sanitária Resolução RDC n. 17, de 16 de abril de 2010. Dispõe sobre as Boas Práticas de Fabricação de Medicamentos.

8. Bermudez J. Medicamentos Genéricos: Uma Alternativa para o Mercado Brasileiro. Cad. Saúde Pública. 1994; 10(3): 368-378. DOI: 10.1590/S0102$311 X 1994000300016$.

9. Barros JAC. Pensando o processo saúde doença: a que responde o modelo biomédico? Saúde e Sociedade. 2002; 11(1): 67-84. DOI: 10.1590/S010412902002000100008.

10. Araújo LU, et al. Medicamentos genéricos no Brasil: panorama histórico e legislação. Rev Panam Salud Publica. 2010; 28(6): 480-492. Disponível em: https://scielosp.org/article/rpsp/2010.v28n6/480-492/pt/

11. Pontes Junior DM, et al. Definition of priority medicines for monitoring laboratory quality in Brazil: the interface between health surveillance and the National Drug Policy. Cad. de Saúde Pública. 2008; 24(9): 2081-2090. DOI: 10.1590/S0102-311X2008000900014

12. Gadelha P, Carvalho JND, Pereira TR. A saúde no Brasil em 2030:

diretrizes para a prospecção estratégica do sistema de saúde brasileiro. Rio de Janeiro: Fiocruz/lpea/Ministério da Saúde/Secretaria de Assuntos Estratégicos da Presidência da República, 2012. Disponível em: https://saudeamanha.fiocruz.br/wp-content/uploads/2016/07/saude2030livro_0.pdf 
13. Biossimilares made in Brazil. Laboratórios prometem cópias de drogas biológicas conhecidas, e oncologistas exigem prova de segurança e eficácia. Rede Câncer. [citado em 05 jul. 2017]. Disponível em: https://www.inca.gov.br/sites/ufu.sti.inca.local/files//media_root/rrc-22assistencia-biossimilares-made-in-brazil.pdf

14. Gonçalves J., et al. Posicionamento da Associação Portuguesa de Farmacêuticos Hospitalares para os biossimilares dos anticorpos terapêuticos. Rev Port Farmacoter. 2015; 7(1):45-52. Disponível em: https://www.apfh.pt/xFiles/scContentDeployer_pt/docs/articleFile229.pdf

15. Maciel V. Ministro quer ações para a competitividade da indústria farmacêutica. portalsaude.saude.gov.br [Internet]. Publicado em 29 de junho de 2016. Disponível em:

http://portalsaude.saude.gov.br/index.php/cidadao/principal/agenciasaude/24342-ministro-quer-acoes-para-a-competitividade-da-industriafarmaceutica.

16. Araújo RF, Andrade VL, Rolim HML. Avaliação do Registro de Medicamentos Nanotecnológicos. Boletim Informativo Geum. 2014; 5(4):46-51.

17. Brasil. Agência Nacional de Vigilância Sanitária. Resolução para regulamentação da notificação, identificação e qualificação de produtos de degradação em medicamentos Resolução RDC n. 53 de 4 de dezembro de 2015. Diário Oficial da União, Seção I, pag 53, 08 de dezembro de 2015.

18. United States Pharmacopeia: USP40 NF36. Rockville: United States Pharmacopeial Convention, Capítulos 232 e 233, 2018.

19 International Conference on Harmonisation- ICH. Guideline for elemental impurities, Q3D. dez, 2016. Disponível em:

https://www.ich.org/fileadmin/Public_Web_Site/ICH_Products/Guidelines/Qualit y/Q3D/Q3D_Step_4.pdf

20. Farias FF, et al. Contributions of Laboratory Results to Promote the Sanitation of the Pharmaceutical Market in Brazil. Lat. Am. J. Pharm. 2011; 30 (3): 502-506.

21 Yano HM, et al. Avaliação da qualidade de formulações farmacêuticas contendo sulfato ferroso, dispensadas na rede pública de saúde. InfarmaCiências Farmacêuticas. 2016; 28(2):75-80.

DOI: http://dx.doi.org/10.14450/2318-9312.v28.e2.a2016.pp75-80

22 Markman BEO, Koschtschak MRW, Auricchio MT. Otimização e validação de método farmacopeico para verificar possíveis desvios de qualidade de matérias primas e cápsulas manipuladas contendo hormônios tireoidianos. Rev Inst Adolfo Lutz. 2007; 66(3): 268-274. Disponível em:

http://periodicos.ses.sp.bvs.br/scielo.php?script=sci_arttext\&pid=S007398552007000300009\&lng=pt\&nrm=iso

23. Gadelha CAG, Vargas MA, Maldonado JMDS, Barbosa PR. O Complexo Econômico-Industrial da Saúde no Brasil: dinâmica de inovação e implicações 
para o Sistema Nacional de Inovação em saúde. Revista Brasileira de Inovação. 2013; 12 (2):251-282. DOI: http://dx.doi.org/10.1590/S003489102012005000065

24. Lanças FM. A Cromatografia Líquida Moderna e a Espectrometria de Massas: finalmente "compatíveis"? Scientia Chromatographica. 2009; 1(2):3561. DOI: http://doi.editoracubo.com.br/10.4322/sc.2013.005

Recebido: 10 de janeiro de 2019. Aceito: 18 de março de 2019

Correspondência: Fernanda Fernandes Farias. E-mail:

farmafernanda@gmail.com

Conflito de Interesses: os autores declararam não haver conflito de interesses.

(C) This is an Open Access article distributed under the terms of the Creative Commons Attribution License, which permits unrestricted use, distribution, and reproduction in any medium, provided the original work is properly cited 